\title{
Synthetic high-resolution line spectra of star-forming galaxies below $1200 \AA$, based on FUSE spectral libraries of hot stars
}

Carmelle Robert ${ }^{1}$, Anne Pellerin ${ }^{1}$, Alessandra Aloisi ${ }^{2}$, Claus Leitherer ${ }^{3}$, Charles G. Hoopes ${ }^{2}$, and Timothy M. Heckman ${ }^{2}$

${ }^{1}$ Département de physique, Université Laval and Observatoire du mont Mégantic, Québec, QC, G1K 7P4, Canada

${ }^{2}$ Department of Physics and Astronomy, The Johns Hopkins University, 3400 North Charles Street, Baltimore, MD 21218, USA

${ }^{3}$ Space Telescope Science Institute, 3700 San Martin Drive, Baltimore, MD 21218, USA

\begin{abstract}
We have generated far-UV stellar libraries using spectra of hot stars in the Galaxy and the Large and Small Magellanic Clouds. These libraries were implemented into the stellar population synthesis codes STARBURST 99 and LAVALSB and used to compute synthetic spectra of star-forming galaxies. Model spectra for galaxies are presented and variations of the hot star photospheric and wind profiles are discussed. This poster summarizes the work of Robert et al. (2002).
\end{abstract}

\section{FUSE spectral libraries}

Four far-UV libraries of $\mathrm{OB}$ and WR stars have been created from the large data pool of the Far-Ultraviolet Spectroscopic Explorer (FUSE) satellite. The FUSE atlasses of hot stars in the Galaxy and Magellanic Clouds by Pellerin et al. (2002) and Walborn et al. (2002) describe the absorption lines and P-Cygni profiles formed in hot star atmosphere and winds.

The solar metallicity library contains 168 stars (WR, O3 to B3). The LMC $\left(\sim 0.3 \mathrm{Z}_{\odot}\right)$ and $\mathrm{SMC}\left(\sim 0.1 \mathrm{Z}_{\odot}\right)$ libraries include 50 and 34 stars (WR, O3 to B0), respectively. A hybrid LMC+SMC library was also created where the $\mathrm{H}_{2}$ contribution from the Magellanic Clouds was removed based on the work of Tumlinson et al. (2002). The libraries cover the wavelength range from 1000.3 to $1182.7 \AA$ (using data from the $\mathrm{SiC} 1 \mathrm{~A}$ and $\mathrm{LiF} 2 \mathrm{~A}$ detectors) with a resolution of $0.127 \AA$, and $\mathrm{S} / \mathrm{N} \simeq 30$ per resolution element. In the libraries, stars with the same spectral type calibration have been combined to increase the $\mathrm{S} / \mathrm{N}$ and an interpolation in temperature or luminosity has been done to create important missing spectral groups.

\section{Synthetic spectra for star-forming regions}

The far-UV libraries have been implemented in the population synthesis codes STARBURSTS99 (Leitherer et al. 1999) and LAVALSB (Dionne \& Robert 2002). 


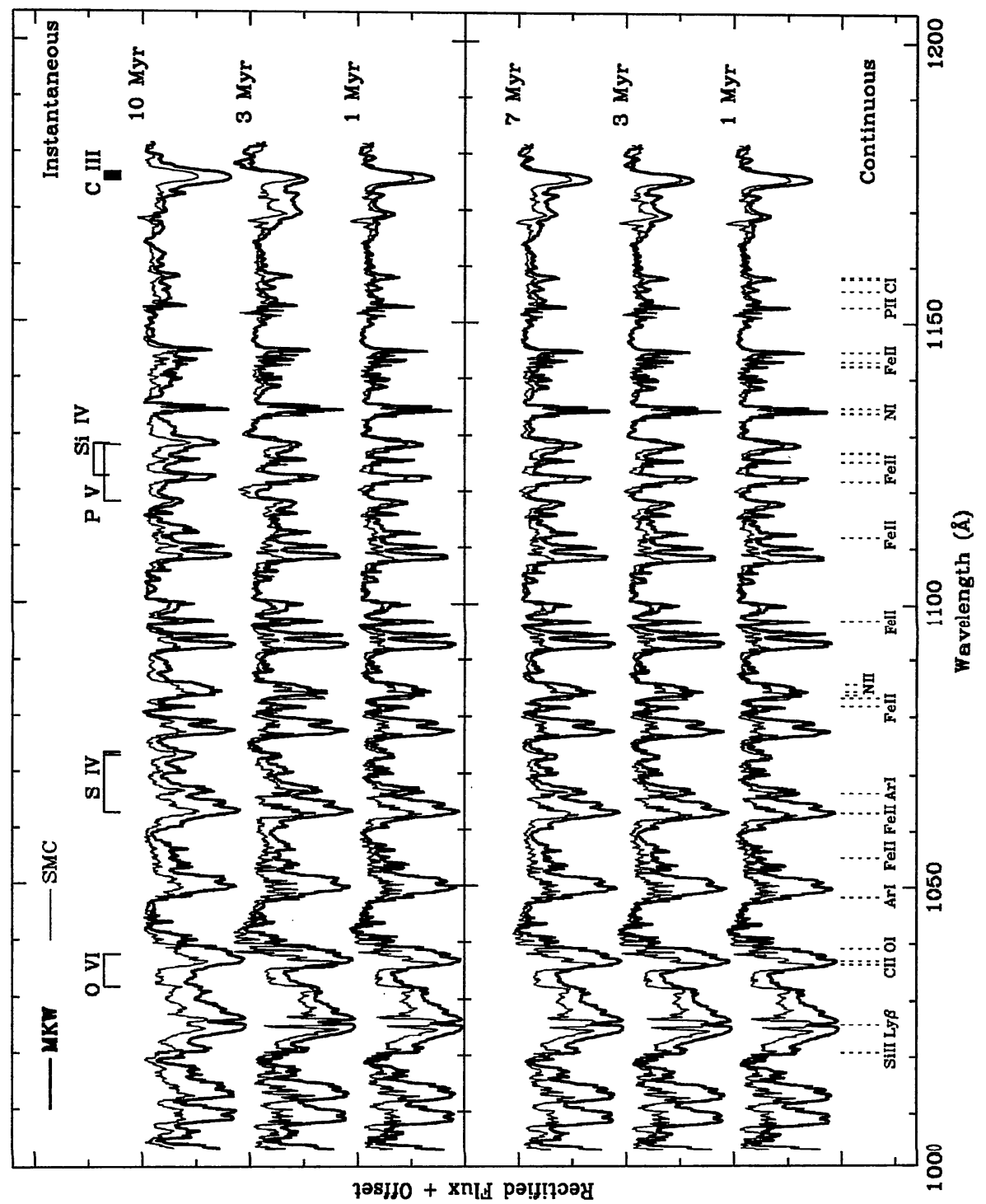

Figure 1. Instantaneous and continuous burst of star formation. Models using a Salpeter. IMF ( $\alpha=2.35)$ with the cut-off masses $M_{l o w}=1 \mathrm{M}_{\odot}$ and $M_{u p p}=100 \mathrm{M}_{\odot}$ are shown for two metallicities (Milky Way: thick lines; Small Magellanic Cloud: thin lines) at three ages. The most important wind lines from hot stars are indicated at the top. Interstellar lines (but not from $\mathbf{H}_{2}$ ) are indentified at the bottom.

Stars form according to a specified star-formation rate and initial mass function (IMF). The stellar evolution is defined by the Geneva groups tracks (prior to rotation). Spectra from a library, at the corresponding metallicity of the tracks, 
are assigned based on the $T_{\text {eff }}$ and $\log g$ calibration of Schmidt-Kaler (1982). Featureless stellar atmosphere models (from Lejeune, Cuisinier \& Buser 1997, and Schmutz, Leitherer \& Gruenwald 1992) are used to flux calibrate the library spectra and to represent missing spectra of cooler stars.

Figure 1 shows synthetic far-UV spectra for various bursts of star formation. Strong wind profiles of C III $\lambda 1176$, O vI $\lambda \lambda 1032,1038, \mathrm{P}$ v $\lambda \lambda 1118,1128$, and S IV $\lambda \lambda 1073,1074$ are predicted when $O$ supergiants stars are present in the stellar population, i.e., $\sim 3 \mathrm{Myr}$. These wind signatures are stronger at higher metallicity. In the instantaneous cases, the wind profiles quickly disappear after $5 \mathrm{Myr}$, when photospheric absorption from B-type stars dominate the models. Because the solar metallicity library do not include stars cooler than B3, the lines predicted for an instantaneous star-forming region are not useful to determine the age when the region is older than $\sim 15 \mathrm{Myr}$. At subsolar metallicity, the libraries do not include stars cooler than B0, therefore the line models for an instantaneous star formation are only useful before $\sim 10 \mathrm{Myr}$. When stars form continuously, an equilibrium between stellar birth and death is reached at $\sim 10$ and $100 \mathrm{Myr}$ for O- and B-type stars, respectively. Continuous models at solar metallicity are all identical after $\sim 10 \mathrm{Myr}$. Without B-type star spectra in the subsolar library, line models for an age $>10 \mathrm{Myr}$ are not useful anymore to reveal the age of a stellar population. For ages greater than the library completeness limit, only the theoretical atmosphere models are available to produce at least a correct absolute value of the far-UV flux. Not shown here, the IMF slope and upper-mass limit also influence the line profile at young ages.

The far-UV spectroscopic features from hot stars can provide important clues on the star-formation history of local and high-redshift galaxies.

\section{References}

Dionne, D., Robert, C. 2002, in preparation

Leitherer, C., Schaerer, D., Goldader, J. E., González Delgado, R.M., Robert, C., Kune, D.F., de Mello, D.F., Devost, D., Heckman, T.M. 1999, ApJS 123, 3

Lejeune, Th., Cuisinier, F., Buser, R. 1997, A\&AS 125, 229

Pellerin, A., Fullerton, A.W., Robert, C., Howk, C., Hutchings, J.B., Walborn, N.R., Bianchi; L., Crowther, P.A., Sonneborn, G. 2002, ApJS 143, 159

Robert, C., Pellerin, A., Aloisi, A., Leitherer, C., Hoopes, C.G, Heckman, T.M. 2002, ApJS 144, 21

Schmidt-Kaler, Th. 1982, in: K. Schaifers \& H.H. Voigt (eds.), Landolt-Börnstein IV: Numerical Data and Functional Relation Ships in Science and Technology (Springer-Verlag: Berlin), p. 1

Schmutz, W., Leitherer, C., Gruenwald, R. 1992, PASP 104, 1164

Tumlinson, J., Shull, J.M., Rachford, B.L., et al. 2002, ApJ 566, 857

Walborn, N.R., Fullerton, A.W., Crowther, P.A., Bianchi, L.B., Hutchings, J.B., Pellerin, A., Sonneborn, G., Willis, A.J. 2002, ApJS 141, 443 